\title{
CERROS ISLA EN UNA CAPITAL LATINOAMERICANA Oportunidad de planificación ecológica y elementos para su diseño
}

\author{
Island hills in a Latin American capital city \\ Opportunities for ecological planning and elements for their design
}

\author{
Picon, María Catalina \\ catalina@santiagocerrosisla.cl \\ Barrera, Francisco \\ Universidad de Concepción \\ fdelabarrera@gmail.com
}

\section{RESUMEN}

Santiago de Chile presenta problemas de dotación y accesibilidad a áreas verdes. El contar con grandes parques urbanos homogéneamente distribuidos ha sido objeto de esfuerzos, no obstante, los nuevos parques son cada vez más pequeños. El presente trabajo busca 1- argumentar la oportunidad que ofrecen los cerros isla de Santiago para resolver inequidades asociadas a las áreas verdes, 2- reconocer y describir su rol ecológico-urbano, y 3- proponer aspectos de diseño que enriquezcan social y ecológicamente a los cerros. Sus grandes extensiones y características geográficas y ecosistémicas posibilitan la provisión de múltiples servicios ecosistémicos. Los cerros isla pueden resolver de manera relevante el déficit en áreas verdes y los principales problemas ambientales de la ciudad si es que son bien diseñados. La ciudad seguirá creciendo, pero tiene que hacerlo de manera adecuada y los cerros isla son estratégicos para incrementar su sustentabilidad y resiliencia.

Palabras clave: desarrollo sustentable, planificación territorial, parques urbanos, mosaico ecológico-urbano. Bloque temático: espacio público y proyecto urbano en la metrópolis contemporánea

\begin{abstract}
Santiago, Chile's capital city, presents problems on the provision and accessibility to open spaces. Efforts to provide large homogeneously distributed urban parks within the city have not been enough, yet, new urban parks are getting smaller. First, this work argues about the opportunity offered by Santiago's island-hills to solve social inequities associated with the accessibility to open spaces. Second, recognize and describe their ecological-urban role. And third, propose design aspects that could enrich the hills in social and ecologically way. Their large extension, and geographic and ecosystemic characteristics, make possible the provision of multiple ecosystem services. Within these regards, if they were well preserved and designed, the island-hills could solve Santiago's deficit of open spaces and main environmental problems. The city will continue to grow; however, it has to do it properly. The island hills are strategic to increase the city's sustainability and resilience.
\end{abstract}

Keywords: sustainable development, territorial planning, urban parks, ecological-urban mosaic. Topic: Public Space and Urban Projects in The Contemporary Metropolis 


\section{Introducción}

La ciudad de Santiago destaca por tener un paisaje y geografía imponente donde resalta la omnipresente y a veces blanca Cordillera de los Andes por el oriente y la acogedora Cordillera de la Costa hacia el poniente. Los ríos Mapocho y Maipo cruzan el valle-en donde se encuentra emplazada la ciudad-de oriente a poniente hasta unirse y desembocar en el mar. En este mismo valle se alzan accidentes geomorfológicos que sobresalen por sobre la trama urbana y en los que todavía se pueden encontrar trazos de ecosistemas naturales; estos últimos se conocen como Cerros Isla. Esta variedad paisajística y geográfica facilita la presencia de una biodiversidad altamente rica y endémica, haciendo de esta zona un hotspot de biodiversidad (Myers et al. 2000).

Santiago, como ciudad capital de un país centralista y en vía de desarrollo, ha experimentado un crecimiento acelerado y condicionado a la economía no solo local o regional, sino también nacional. Este aspecto económico ha permeado en la planificación urbana de los últimos 40 años, estableciéndose una predominancia del uso del paisaje y geografía como recursos que pueden generar réditos económicos, disminuyendo así el reconocimiento de sus papeles ecológicos o ambientales y generando un impacto negativo sobre el paisaje; especialmente en términos de su biodiversidad y sus suelos (Montoya-Tangarife et al. 2017). Pese a esto, lo que queda del paisaje ofrece aún la oportunidad de redireccionar la planificación territorial para así incorporar más criterios basados en la riqueza geográfica y paisajística, utilizando enfoques asociados a la planificación ecológica o sustentable del territorio.

El futuro de la ciudad de Santiago será lo que sus habitantes y, en especial, lo que sus tomadores de decisiones o actores claves decidan. Para esto es importante plantear visiones, criterios generales, y plasmarlos en los instrumentos de planificación existentes. En el pasado han existido instrumentos que han destacado estos aspectos paisajísticos y naturales, estableciendo zonas de protección ecológica en los cordones montañosos circundantes a la ciudad, restringiendo el uso de las cajas de los ríos y reconociendo zonas de riesgos naturales. Sin embargo, las debilidades normativas locales y regionales han favorecido un rápido desarrollo urbano donde los instrumentos asociados a la protección y/o incorporación de elementos naturales en la ciudad han sido insuficientes. Muchos sitios con estos elementos se encuentran en condiciones de deterioro, e incluso, urbanizados. Tal es el caso de los cerros isla urbanos, que fueron inicialmente declarados como "de utilidad pública" por los instrumentos de planificación-ya sea en su totalidad o parte de ellos-. No obstante ello, el año 2004 se modifica el artículo 59 de la Ley General de Urbanismo y Construcciones (LGUC), con el objeto de establecer la caducidad de declaratoria en los planes reguladores (Ley $n^{\circ} 19939$ del Ministerio de Vivienda y Urbanismo); esta modificación afectó los cerros isla dejándolos desprovistos de protección ante el desarrollo inmobiliario, principalmente. Posteriormente, el año 2014, el art. 59 de la LGUC fue nuevamente modificado, manteniendo la afectación de utilidad pública a terreno como los Cerros isla (Ley $n^{\circ} 20.791$ del Ministerio de Vivienda y Urbanismo).

En la actualidad Santiago tiene un gran déficit de áreas verdes $\left(3.3 \mathrm{~m}^{2} / \mathrm{hab}\right.$; Cedeus, 2017) índice que se encuentra muy por debajo de lo que otros países o instituciones han fijado como estándares (Wang, 2009; De la Barrera et al. 2016a). Esta problemática se intensifica aún más al identificar que las áreas verdes disponibles en el área urbana están desigualmente distribuidas (Forray et al., 2012, De la Barrera et al. 2016a) y en muchos casos, son lugares de baja calidad y con limitada accesibilidad para las personas, ya que se encuentran aisladas dentro del tejido urbano (Reyes-Paecke \& Figueroa, 2010; De la Barrera et al. 2016a). Dicha accesibilidad es medida en términos de tiempo de caminata hacia cada tipo de área verde, siendo mayor hacia parques que hacia plazas, por lo que es deseable contar con parques homogéneamente distribuidos en la ciudad. Esto último ha sido objeto de esfuerzos por parte del Estado de Chile, existiendo un amplio desarrollo de nuevos parques urbanos, estando ese esfuerzo destinado en Santiago hacia parques de más de 2 hectáreas y mayormente en sectores planos (De la Barrera \& Henriquez 2017).

En este sentido, los objetivos de este trabajo son (1) argumentar la oportunidad de planificación territorial y urbana que ofrecen los cerros isla de la ciudad de Santiago para resolver inequidades en la provisión y el 
acceso a áreas verdes, (2) reconocer y describir su rol como parte de un mosaico ecológico urbano, donde los cerros isla son parte de una infraestructura verde o ecológica, y (3) proponer aspectos de diseño ecológico que deben incorporarse para transformar a los cerros isla en espacios ricos tanto social como ecológicamente. Metodológicamente, se realiza un análisis de información geográfica con el foco de reconocer las partes integrantes del mosaico ecológico urbano desarrollando una integración de capas en Sistemas de Información Geográfico, tras lo cual se presentan las observaciones y conclusiones de un amplio proceso de discusión entre especialistas y actores-involucrados en la planificación urbana y territorial-para evaluar las oportunidades específicas que surgen al considerar los cerros isla como elementos significativos de planificación.

Si bien estos argumentos están desarrollados para la ciudad de Santiago, su aplicación es factible en otras ciudades similares, donde los cordones montañosos rodean y se integran con la ciudad, invitando a observarlos como partes integrales de la ciudad y no como hechos aislados. Esta situación es común tanto en otras regiones de Chile como en ciudades latinoamericanas asociadas a la Cordillera de los Andes.

\section{Cerros isla como oportunidad de planificación}

\subsection{Contexto histórico}

Los incas dieron valor al actual Santiago por su cercanía y vista del Cerro El Plomo, de gran importancia ceremonial para esta cultura prehispánica. Los mapuches también reconocieron, especialmente, el potencial de uno de los cerros isla, el cerro Huelén, actual Santa Lucía, usándolo como sitio ceremonial y cementerio. Luego los españoles, a través de Pedro de Valdivia, visualizaron las ventajas de fundar Santiago como asentamiento en un valle fértil cruzado por un río y con cerros que permitirían su seguridad. Ya en el siglo XIX, el entonces intendente Benjamín Vicuña Mackenna vio en uno de estos mismos cerros, el Cerro Santa Lucía, una gran oportunidad para crear un gran pulmón verde para la ciudad y decide transformarlo en el Paseo del Santa Lucía, una "plaza aérea" de uso público, "un espacio de recreo y arte, salud e higiene" para poner en valor el patrimonio natural e histórico del cerro (Vicuña Mackenna, 2014). Siguiendo la misma lógica, un siglo después el intendente Alberto Mackenna inicia el proceso de expropiación del cerro San Cristóbal para transformarlo en el Primer Parque Metropolitano para Santiago.

\subsection{Contexto actual}

El contexto urbano, social y medioambiental actual de la ciudad de Santiago se encuentra muy alejado de un desarrollo sustentable y resiliente, lo que se ha visto reflejado en bajos índices de calidad urbana que se relacionan directamente con la calidad y cantidad de áreas verdes existente en una ciudad.

Los dos cerros nombrados anteriormente-Santa Lucía y San Cristóbal-son solo dos entre los aproximadamente sesenta y dos Cerros Islas existentes en la Región Metropolitana de Santiago. Veintiséis de ellos se encuentran ubicados dentro o adyacentes al límite urbano de la ciudad y/o se encuentran identificados como Cerros Isla en los instrumentos de planificación territorial de la región (Picon \& Ruiz, 2017). Mayoritariamente siguen sin ser urbanizados y, salvo excepciones, no cuentan con intervenciones relevantes. En muchas ocasiones están deteriorados y representan espacios propicios para situaciones no deseadas, tales como actos de delincuencia, propagación de incendios, establecimiento de basurales ilegales, derrumbes, etc.

Dado al gran potencial presente de los Cerros Isla para enfrentar problemáticas relacionadas al déficit y desigual distribución de áreas verdes en la ciudad, la Fundación Cerro Isla, gracias al apoyo de Centro de Políticas Públicas de la Pontificia Universidad Católica de Chile, pública en 2012 una investigación que tuvo como objetivo plantear la integración de los cerros isla al sistema de áreas verde de Santiago, con el objetivo de incluirlo en las políticas públicas nacionales. El persistente trabajo de la fundación por medio de la generación de investigaciones, coordinación de seminarios con expertos en la materia y su constante gestión, 
se resume en su mayor logro: posicionar en la agenda pública regional y local la necesidad de recuperar los cerros isla.

Un gran impulso a este propósito fue el concurso "Iniciativas para Cerros Isla en la Región Metropolitana", convocado por Gobierno Regional de la Región Metropolitana de Santiago (GORE RM), que contó con el apoyo de la Fundación. Este concurso incentivó a los gobiernos locales a consolidar los cerros isla dentro de su territorio como parques urbanos. En la actualidad varios municipios-tales como San Bernardo, Renca, Lo Barnechea, Las Condes y Puente Alto-, están trabajando o bien tienen intenciones serias de consolidar cerros ubicados dentro de su territorio como parques urbanos naturales. En los cerros de Renca (Renca) y Chena (San Bernardo), por ejemplo, los respectivos municipios están desarrollando proyectos de parque en las faldas de los cerros. Ambos proyectos impulsados por el concurso del GORE RM. En los casos del Cerro del Medio (Lo Barnechea), El Calán (Las Condes) y La Ballena (Puente Alto), los gobiernos locales están en el proceso de desarrollar proyectos en cada uno de los cerros. Algunos de estos municipios han contado con el apoyo de la Fundación Cerros Isla, una organización no gubernamental que busca valorizar, integrar y aprovechar los elementos naturales del paisaje para mejorar la calidad de vida de las personas. La fundación se ha propuesto como primera misión recuperar los 26 cerros islas ubicados dentro del área urbana de Santiago para consolidarlos como espacios recreativos de uso público, enfrentado así los desafíos ecológicos, sociales y económicos, relacionados con las problemáticas urbanas y sociales del siglo 21. Estos 26 cerros son: Alvarado, Adasme, Apoquindo, Blanco, Calán, Chena, Chequén, Del Medio, Dieciocho, El Manzano, Hasbún, Jardín Alto, La Ballena, Las Cabras, Lo Aguirre, Loma Larga, Los Morros, Los Piques, Navia, Negro, Renca, Los Almendros, San Cristóbal, San Luis y Santa Lucía (Fig. 1), los cuales se encuentran dentro de la zona urbana de Santiago y afectos a utilidad pública.

A pesar del impulso que están teniendo los gobiernos locales para desarrollar los cerros como espacios públicos, queda un gran trabajo por hacer. Existen muchos otros cerros por integrar como áreas verdes y es de suma importancia asegurar que los proyectos en pleno desarrollo incorporen por medio del diseño, la protección y conservación de estos ecosistemas naturales; para así asegurar que el uso de estos elementos naturales-como espacios públicos-no afecte su estado natural, sino que, al contrario, asegure y favorezca su condición natural en el tiempo. 


\section{SIIU

barcelona|santiago de chile junio 2019

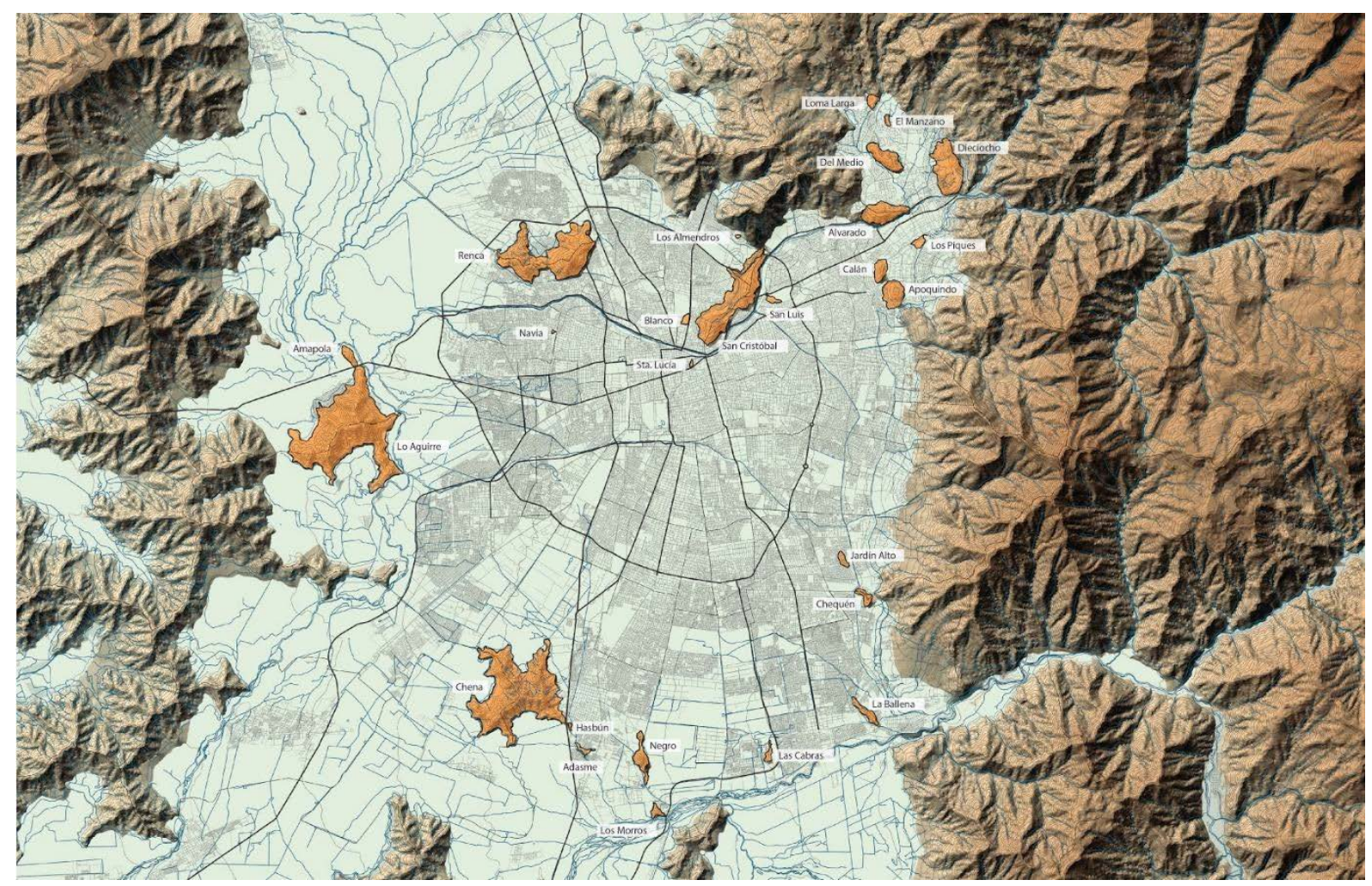

Fig 1. Ciudad de Santiago y su contexto. Fuente: Fundación Cerros Isla.

\subsection{Cerros isla como áreas verdes}

Las áreas verdes ya sea urbana o naturales aportan grandes beneficios a los habitantes de las ciudades. Estudios indican que en términos ambientales pueden ayudar a disminuir las temperaturas en las ciudades, limpiar el aire, aportan a la infiltración de aguas lluvias, entre muchos otros servicios ecosistémicos (Chiesura et al 2004, Tyrvainen et al. 2005, Dobbs et al. 2014, De la Barrera et al. 2016b). En términos sociales, estas zonas de esparcimiento pueden aportar a la salud mental y física, y a la integración social, ya que dan la posibilidad del contacto directo con la naturaleza (De la Barrera et al. 2016c). Este último punto, se puede ver reflejado incluso en el gasto público, ya que las áreas verdes pueden ayudar a evitar múltiples enfermedades que hoy tienen que ser tratadas por el servicio público de salud.

Los cerros isla por su masa crítica y ubicación son considerados un recurso crítico para enfrentar el déficit de áreas verde y su desigual distribución en el territorio; las comunas de bajos recursos apenas llegan a los $3 \mathrm{~m}^{2}$ de áreas verdes por habitante en comparación con las comunas más acomodadas que se acercan a las $9 \mathrm{~m}^{2}$ de áreas verdes por habitante (Picon \& Ruiz, 2017, De la Barrera et al. 2016a). En total, los 26 cerros identificados dentro del área urbana de Santiago suman cerca de 5.000 ha de terrenos disponibles para ser incorporados en el sistema de áreas verdes de Santiago, y así incrementar de 3 a $12 \mathrm{~m}^{2}$ de áreas verdes por habitante (Cedeus 2017; CTT, UAI 2017). Más potente aún, es el hecho de que mucho de estos cerros $-70 \%$ de la superficie total de los cerros (Picon \& Ruiz, 2017)- se encuentran localizados justamente en las comunas más vulnerables y de menores recursos; 15 cerros se encuentran ubicados en comunas donde el índice de calidad de vida es inferior a la media nacional (Forray et. al., \& Orellana et al., 2012). La comuna de San Bernardo, por ejemplo, cuenta con el cerro Negro, Hasbún, Adasme y parte del cerro Chena. Si estos cerros fueran parte del sistema de áreas verde de esta comuna, San Bernardo pasaría de tener $2 \mathrm{~m}^{2}$ a $10 \mathrm{~m}^{2}$ por habitante, acercándose a comunas de altos ingresos como Vitacura que actualmente posee $8 \mathrm{~m}^{2}$ de áreas verdes por habitantes (De la Barrera et al. 2016a). Es por esta razón que los cerros isla constituyen un recurso significativo para aumentar la cantidad de áreas verdes en estas comunas, promoviendo principios de equidad en los procesos de desarrollo urbano y aumentando la calidad de vida de los ciudadanos. 
A la cantidad de áreas verdes hay que añadirle el factor de calidad, el cual se relaciona con el tamaño de estas áreas. En Santiago más del $90 \%$ de las áreas verdes no superan los $5.000 \mathrm{~m} 2$ y en las comunas de menores ingresos, estas tienen un tamaño menor a los 1.000 m2 (Reyes-Paecke \& Figueroa 2010, Forray et. al., 2012). Los cerros isla por su lado presentan superficies que van desde los 2,72 ha (Cerro Navia) hasta los 1.744 ha (Lo Aguirre) $(\mathrm{FCl}, 2017)$, por lo que tienen el gran potencial de contribuir a la calidad de las áreas verdes de la ciudad.

\subsection{Planificación territorial y cerros isla}

Muchos de los cerros isla cubren áreas que están fuera del límite urbano regulado de cada comuna, pertenecen a más de un territorio comunal, y/o están solo zonificados por el instrumento de planificación regional, excluidos del instrumento comunal. En el Plan Regulador Metropolitano de Santiago, varios de los cerros isla -o parte de ellos- están reconocidos como parte del Sistema Metropolitano de Áreas Verdes y Recreación, lo que los excluye del desarrollo urbano.

Los cerros isla, en su mayoría, son hoy elementos aislados dentro de la ciudad debido principalmente a que la planificación no los ha reconocido como parte de un sistema geográfico, natural o ecológico específico, más allá de asignarles un uso de suelo como área verde (Forray et al., 2012). Debido a las debilidades normativas la mayoría de estos cerros no se han podido consolidar como espacios naturales de uso público, transformándose en focos de delincuencia e inseguridad para las comunidades que colindan estos cerros, o bien han quedado totalmente aislados dentro de la trama urbana, quedando fragmentada su relación con el entorno natural, provocando su deterioro y muerte de sus ecosistemas naturales. En varios casos, estas debilidades han favorecido el desarrollo de proyectos inmobiliarios. En otros casos, han dificultado la gestión de los cerros ya que no existe concordancia entre los limites naturales de los cerros y los limites administrativos, donde la división del territorio en comunas en muchos casos frena la consolidación de parques, y la ejecución de mantención cotidiana. Por otro lado, cerca del $80 \%$ de los cerros identificados dentro de Santiago son de propiedad privada, lo que también genera dificultades adicionales para su gestión y consolidación como zonas públicas. Hasta el momento, todos los cerros que están en proceso de consolidación son de tenencia pública, siendo fundamental el liderazgo y el compromiso de las autoridades locales y regionales. Berrizbeitia (2017), explica que un gran obstáculo a superar para lograr la conservación de estos cerros es alcanzar el diseño y la implementación de procesos y mecanismos-legales y económicos-que incentiven la consolidación de los cerros como parques. También es importante lograr que el diseño sea el pertinente al paisaje y geografía de los cerros y por ende del territorio. A pesar que existe una tendencia a la protección y consolidación de los cerros islas como áreas naturales públicas, la realidad demuestra lo contrario: los instrumentos de planificación tienden a facilitar el desarrollo urbano en los cerros más que protegerlos y consolidarlos como zonas naturales urbanas.

Los cerros islas son parte de los elementos naturales esenciales de la ciudad, cuando se piensa el desarrollo urbano desde una perspectiva ecológica (Picon et. al., 2017). A pesar de que estos ecosistemas naturales, hoy se encuentran con un alto nivel de degradación, todavía brindan la valiosa oportunidad de hacer de Santiago una ciudad más resiliente y sustentable. Lo anterior podría ser posible si es que éstos se restauraran y se conservaran, y si estuvieran mejor incorporados dentro de las normativas de planificación (Picon et al., 2017). Asimismo, si se considera la proximidad de estos cerros a los cursos de agua presentes en el valle, su relación con los cordones montañosos de las cordilleras de la Costa y de los Andes, y las áreas verdes existentes en la ciudad, se podría conformar un sistema de elementos naturales y urbanos integrados y conectados, conformando una matriz urbana y ecológica -infraestructura verde o ecológica- que potencie el funcionamiento ecológico y social de estos cerros (Picon \& Ruiz, 2017).

A pesar que alguno de estos o parte de ellos se encuentren protegidos bajo zonas de utilidad pública, y por ende debiesen estar abocados a ser parques urbanos, la mayoría de ellos se encuentran en una constante presión urbana en cuanto al cambio de su uso de suelo (Forray et. al., 2012). Los cerros que se encuentran en las zonas de más altos ingresos, se encuentran también bajo presión inmobiliaria, lo que se ve reflejado en los 
paños de suelo aledaños a los cerros que superan las 30UF/m² (Forray et al., 2012). En consecuencia, a esta presión, la urbanización ha ido remontando los cerros en algunos casos y en otros casos dejándolos aislados. En ambos casos, las intervenciones han sido altamente agresivas afectando su condición natural, disminuyendo su biodiversidad y por ende aumentando su erosión. Esta situación se da principalmente en los cerros ubicados en el sector nororiente: Alvarado (Lo Barnechea), Dieciocho (Lo Barnechea), Del Medio (Lo Barnechea), Los Piques (Las Condes), Calán (Las Condes), Apoquindo (Las Condes), y San Luis (Las Condes). Por otro lado, cerros ubicados hacia el sur de la ciudad como el cerro Jardín Alto (La Florida) se encuentran construidos casi en su totalidad, y otros como el Cerro Las Cabras (Puente Alto) donde sus alrededores se han utilizado para extracción de áridos. En la mayoría de los casos el encuentro de la trama urbana con la parte baja de los cerros no ha sido bien resuelta (Forray et. al., 2012), la manera en cómo se aborda este encuentro denota una falta de sensibilidad e ignorancia de la importancia ecológica de estos elementos para el bienestar de todos los habitantes, provocando su deterioro y convirtiéndolos en lugares poco accesibles, habitables y especialmente inseguros para la comunidad.

En este sentido, es necesario promover la desestigmatización de estos ecosistemas naturales transformando la percepción negativa que se tiene de muchos de ellos, pasando de ser focos de basura y delincuencia a espacios públicos de calidad, mejorando así la calidad de vida de los habitantes de la ciudad. Tal como lo propone la Fundación Cerros Isla "es necesario capitalizar lo existente aunando las facultades y responsabilidades tanto de los organismos públicos como privados."

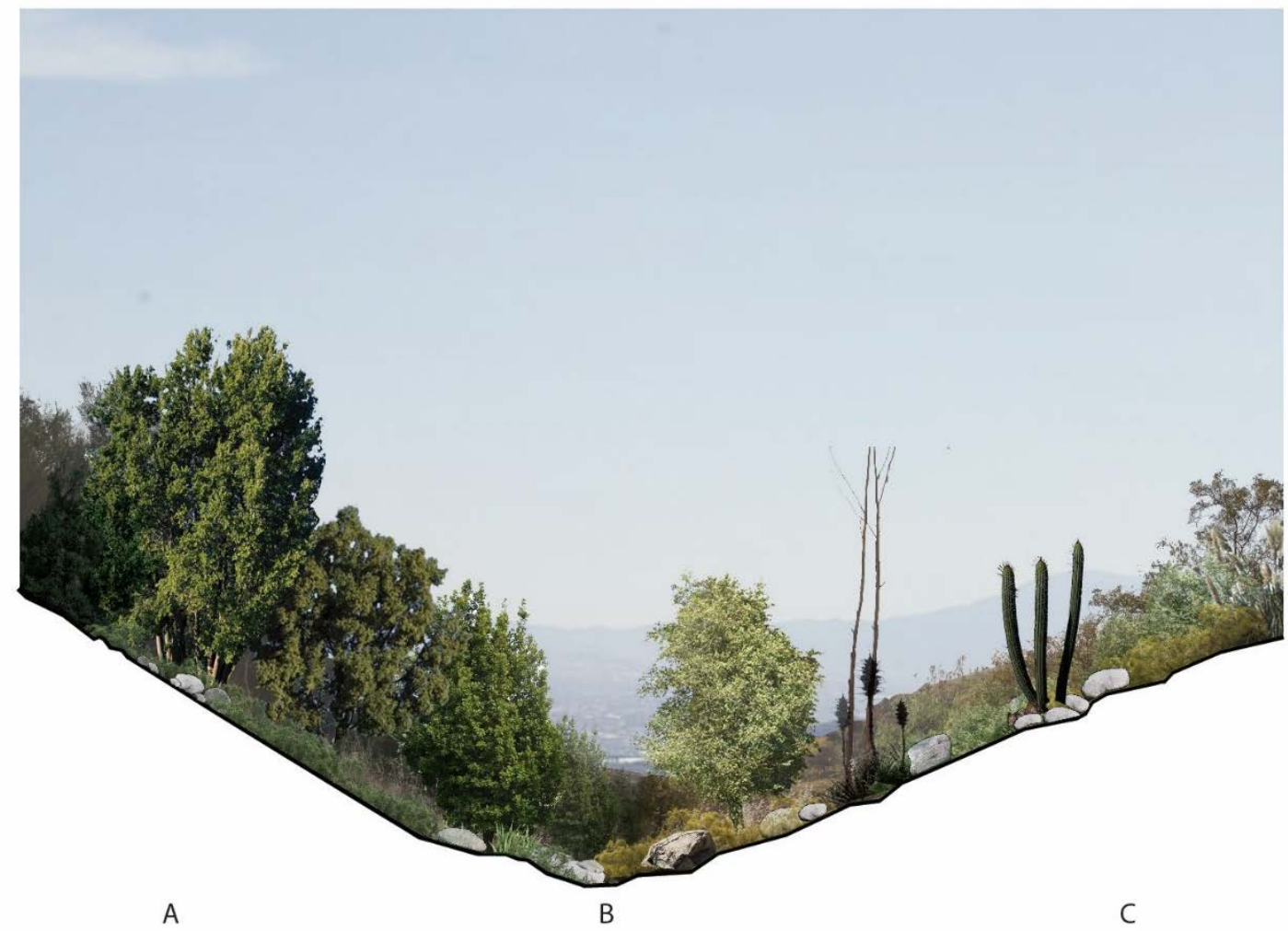

Figura 2. Distribución tipo de los pisos de vegetación en un cerro isla. Donde: A: representa a la ladera sur, estando compuesta por Litre, Peumo y Quillay; B: quebrada (interzonal), con especies como Belloto y Quillay; y C: ladera norte, con Madreño, Puya, Cactus, Huañil y Cola de Zorro. Realizado por FCl en base a: Luebert, F., \& Pliscoff, P. (2006) Sinopsis bioclimática y vegetacional de Chile. 


\section{Rol de los cerros isla en un mosaico ecológico urbano}

Comprender la ciudad como un mosaico ecológico urbano implica el reconocer las estructuras y funciones principales de los componentes naturales o seminaturales que rodean la ciudad y que están dentro de ella, sea porque son remanentes de la naturaleza que antes dominaba el paisaje o bien porque fueron implementadas como áreas verdes en un proceso de planificación (Picon et al. 2017). Este reconocimiento tiene por objetivo no solo identificarlas como objetos a conservar o excluir del desarrollo urbano, sino que entenderlas como parte integral del espacio urbano, que requiere de estos elementos para interactuar con ellos, por las múltiples funciones que ofrece (Picon et al. 2017). Un mosaico ecológico urbano es un sistema integrado entre elementos construidos y elementos naturales que conforman lo que denominamos ciudad. Este concepto urbano busca incorporar en la planificación urbana a los ecosistemas naturales como parte integrante del sistema a planificar, sea cual sea su escala, con el fin de asegurar su pleno funcionamiento como sistema ecológico y evitar la disociación entre la naturaleza y la sociedad, que ha llevado a la crisis ambiental actual. Para lograr esto, se trabaja con un enfoque multiescalar-macro-regional, regional y urbano-con el objetivo de entender en su totalidad el territorio a planificar. Se utiliza el modelo de "parches, corredores y matriz" propuesto por Richard Forman, en relación a la detección y articulación de elementos, velando por incrementar la conectividad de los flujos ecológicos. Estos principios de la ecología del paisaje y urbana dan luces sobre la importancia de entender la ciudad y su entorno como un sistema en donde todo se encuentra conectado, lo que permite a estos centros urbanos lograr autosustentarse y permanecer en el tiempo.

Los cerros isla, llevan el nombre de isla porque están rodeados de algo que es diferente a lo que tiene el cerro mismo: no se trata de agua como en una isla verdadera, sino que, de la ciudad tradicionalmente gris, sellada y sin vegetación. No obstante, la ciudad puede ser diferente, y el encuentro entre la trama urbana y los cerros isla puede ser menos drástico. Más adelante se ofrecen algunas alternativas para promover este enfoque. Los cerros isla tienen como característica el ser espacios amplios (hasta $1.744 \mathrm{ha}$ ) por lo que, si son implementados adecuadamente, pueden representar grandes parques urbanos, pero distintos a los parques tradicionales, $y$ tal como lo muestran los cerros islas que han sido desarrollados como parques, representan parques urbanos naturales, que requieren poca mantención.

\subsection{Beneficios ecosociales}

En un mosaico ecológico urbano, un cerro isla con vegetación bien conservada, e infraestructura adecuada para acceder a ellos y utilizarlos, representa un elemento capaz de proveer múltiples funciones y/o servicios ecosistémicos, es decir beneficios aportados por la naturaleza hacia las personas. Algunos de estos servicios ecosistémicos son: ofrecer espacios para la recreación, la cohesión social, la educación formal e informal, la reflexión o espiritualidad, la identidad y la seguridad frente a desastres naturales; servir como espacios para la regulación del agua en términos de infiltración, escorrentía y humedad del aire, mitigar las altas temperaturas en verano, capturar contaminantes atmosféricos locales y carbono, contener especies valiosas para la polinización, la contemplación y la dispersión de semillas; y permitir el crecimiento y extracción sustentable de frutales, plantas medicinales, recursos madereros o del suelo, la captura y cosecha de agua, entre muchos otros servicios. Los cerros isla tienen el gran potencial de poder ser los nuevos pulmones verdes como también lugares de esparcimiento de gran calidad para los habitantes de ciudades montañosas.

\section{Diseño ecológico en cerros isla}

Es esencial planificar y diseñar reconociendo las estructuras y funciones principales de estas áreas naturales. En este sentido, los cerros se diferencian de los parques y plazas urbanas por ser lugares que no son planos, sino que tienen pendientes de hasta $45^{\circ}$ grados, por lo que deben ser entendidos y por ende diseñados considerando esta condición. Esta variedad de pendientes en los cerros genera limitaciones, como también oportunidades que se debieran tener en cuenta al momento de consolidar estos lugares como espacios naturales de esparcimiento. 
En primera instancia es de suma importancia resolver el encuentro de la trama urbana con la ciudad. En la actualidad no existe un perfil de calle que se adapte a este encuentro ni un diseño claro que resuelva este tipo de situación, lo que parece difícil de comprender, considerando que Santiago se encuentra emplazado en un valle que se encuentra rodeado de cordones montañosos que conforman un $75 \%$ de la superficie de la población (www.sinia.cl). Esto ha generado grandes cortes en los cerros y en quebradas de alto valor medioambiental que se han visto deteriorados, como también convertidos en micro-basurales, y en muchos casos, en lugares inseguros (Forray et al., 2012).

La pendiente de los cerros puede, por un lado, dificultar el uso de estos lugares, pero también puede enriquecer los hábitats naturales presentes en estos ecosistemas naturales. Por sólo el hecho de tener distintos tipos de pendientes el grado de erodabilidad, de vegetación y la accesibilidad van a variar. Incluso la manera de cómo se comporta el agua que cae con las precipitaciones, tras caer sobre el suelo e infiltrarse o fluir superficialmente, en modo de escorrentía por sus faldeos. O incluso, la manera en que el sol irradia a estos promontorios va a variar según el grado de pendiente y ubicación en relación al sol. Esto define el tipo de vegetación que crece en los cerros, siendo diferente si se encuentra expuesta a los rayos del sol (solana) o si está permanentemente sombreada y húmeda (umbrías).

El grado de pendiente también debiese definir el tipo de uso social o programa que se puede dar en lugares específicos en los cerros. Es decir, es más probable que más personas puedan acceder a las partes de menor pendiente y menos personas puedan acceder a los lugares con mayor pendiente; lo mismo con las quebradas, ya que son lugares más difíciles de llegar. Es más realista pensar que los cerros se puedan habitar en tan sólo los lugares más accesibles y de menor valor medioambiental, protegiendo al mismo tiempo hábitats naturales dentro el mismo ecosistema del cerro que son esenciales para preservar su condición natural y enfrentar problemáticas asociados a la erosión y falta de flujos de fauna. Esta situación permite una regulación orgánica de los lugares que se quieran conservar y/o preservar en los cerros.

Al momento de pensar los cerros como parques urbanos naturales para la ciudad, es imprescindible comprender su estructura ecológica, lo que va a asegurar su sustentabilidad y resiliencia en el tiempo. Es decir, que es necesario permitir que se sigan desarrollando los flujos naturales que se dan en estos lugares con otros ecosistemas dentro del valle. También es importante conservar los lugares de mayor valor medioambiental como lo son las quebradas. Se deben considerar en su diseño los atributos naturales que se expresan en estos cerros como son las escorrentías, las zonas más o menos sombrías, su conexión o relación con cursos hídricos, cerros, $u$ otras zonas naturales que pueden enriquecer su biodiversidad. Es necesario comprender que este tipo de consideraciones pueden ayudar a facilitar e incluso disminuir los gastos de mantención de la vegetación de estos lugares.

Siguiendo estas directrices es necesario resolver cómo es el encuentro de los cerros con la trama urbana. Este encuentro no debiese representar un límite abrupto o arbitrario que sea definido a partir de un punto o altura específica, sino que más bien, debería ser una zona que se adapta a los límites y atributos naturales del cerro y a la relación del valle y la ciudad, generando así una relación en forma de gradiente, que va desde una situación más natural (asociado al cerro) hasta una más propia de lo construido (asociado a la ciudad).

Actualmente muchos de estos cerros se encuentran muy degradados en términos ecológicos. Esto significa que es necesario restaurarlos como ecosistemas naturales. Para este caso, es importante destacar que los mecanismos de restauración deben ser los adecuados para conservar el estado natural de los cerros y poder devolverle todas sus propiedades naturales para que puedan seguir otorgándole a la ciudad todos los beneficios ecosistémicos necesarios para nuestra supervivencia. Es necesario facilitar la restauración de la vegetación tanto activa (ej. a través de planes de reforestación, revegetación o nucleación) como pasiva (ej. eliminando perturbaciones), privilegiando la presencia de vegetación nativa propia de cada condición de cada cerro y de sus procesos naturales, ejerciendo así un manejo pasivo de la vegetación. 


\section{Conclusión}

Los cerros islas tienen el potencial de ser un elemento de alto valor ambiental y social en el mosaico ecológicourbano de la ciudad, en el que existen elementos naturales del paisaje que pueden integrarse de manera armónica. En caso contrario, pueden convertirse en espacios inconexos que dan lugar a situaciones de degradación y abandono. La incorporación de los cerros islas al desarrollo urbano, como elementos bien conservados y adecuadamente desarrollados, requiere reconocer los beneficios sociales que genera un buen desempeño ecológico de la naturaleza contenida en ellos. Por eso la implementación de éstos como parques urbanos naturales requiere de elementos de diseño que reconozcan su naturaleza y características geográficas. La ciudad seguirá expandiéndose, por lo que es imperativo planificar su conservación y habilitación como áreas verdes especiales, que contribuyan a hacer de la ciudad un lugar más sustentables y resilientes que puedan enfrentar las problemáticas asociadas al cambio climático y a ciudades en vías de desarrollo.

\section{BIBLIOGRAFÍA}

BERRIZBEITIA, A. (2017). Conclusión: Los cerros isla y la dimensión pública del paisaje. En: FUNDACIÓN CERROS ISLA. Cerros Isla de Santiago, Construyendo un nuevo imaginario de ciudad a partir de su geografía. Ediciones ARQ, Santiago.

CENTRO DE DESARROLLO URBANO SUSTENTABLE (CEDEUS) (2017). Indicadores de sustentabilidad urbana. Aplicación en las 6 ciudades CEDEUS. Informe ejecutivo.

CENTRO DE INTELIGENCIA TERRITORIAL, UAI. (2012). Plano de estratos socio económicos por quintil de Santiago, basado en CASEN 2011.

CHIESURA, A. (2004). The role of urban parks for the sustainable city. Landsc. Urban Plan. 68 (1), 129-138.

DE LA BARRERA, F. y HENRÍQUEZ, C. (2017). Monitoring the Change in Urban Vegetation in 13 Chilean Cities Located in a Rainfall Gradient. What is the Contribution of the Widespread Creation of New Urban Parks? Materials Science and Engineering 245(7). doi:10.1088/1757-899X/245/7/072023

DE LA BARRERA, F., REYES-PAECKE, S. y BANZHAF, E. (2016a). Indicators for green spaces in contrasting urban settings. Ecological Indicators, 62, 212-219.

DE LA BARRERA, F., RUBIO, P. y BANZHAF, E. (2016b). The value of vegetation cover for ecosystem services in the suburban context. Urban forestry \& urban greening, 16, 110-122.

DE LA BARRERA, F., REYES-PAECKE, S., HARRIS, J., BASCUÑÁN, D. FARÍAS, J.M. (2016c). People's perception influences on the use of green spaces in socio-economically differentiated neighborhoods. Urban Forestry \& Urban Greening, 20, 254-264.

DOBBS, C., KENDAL, D. y NITSCHKE, C.R. (2014). Multiple ecosystem services and disservices of the urban forest establishing their connections with landscape structure and sociodemographics. Ecol. Indic. 43, 44-55

FORRAY, R., ARELLANO, E., PICON, C., FERNÁNDEZ, I., BESA, A., RUIZ, F. y LEFRANC, E. (2013). Plan for integrating Santiago's Island-hills into its system of green spaces. In: Centro de Políticas Públicas UC ed. Concurso Políticas Públicas 2012, Propuestas para Chile. Santiago: Pontificia Universidad Católica de Chile, 177-209.

FUNDACIÓN CERROS ISLA (2017). Cerros Isla de Santiago, Construyendo un nuevo imaginario de ciudad a partir de su geografía. Ediciones ARQ, Santiago. 
MACKENNA, B.V. (1874). Album del Santa Lucia: colección de las principales vistas, monumentos, jardines, estatuas, y obras de arte de este paseo, dedicado a la Municipalidad de Santiago por su actual presidente Benjamín Vicuña Mackenna. Imprenta de la Librería del Mercurio de A. y M. Echeverria, calle de Morandé, núm. 38.

MONTOYA-TANGARIFE, C., DE LA BARRERA, F., SALAZAR, A. y INOSTROZA, L. (2017). Monitoring the effects of land cover change on the supply of ecosystem services in an urban region: A study of SantiagoValparaíso, Chile. PloS one, 12(11), e0188117.

MYERS, N., MITTERMEIER, R. A., MITTERMEIER, C. G., DA FONSECA, G. A. y KENT, J. (2000). Biodiversity hotspots for conservation priorities. Nature, 403(6772), 853.

PICON, C. y RUIZ, F. (2017). Los cerros isla: una oportunidad para mejorar la calidad de vida de la ciudad. En Cerros Isla de Santiago, Construyendo un nuevo imaginario de ciudad a partir de su geografía. Ediciones ARQ, Santiago, pp. $14-40$

PICON, C., DE LA BARRERA, F., CONTRERAS, C., BERRIZBEITIA, A., REYES-PAECKE, S. y FERNÁNDEZ, F., (2017). Mosaico Ecológico Urbano: Criterios para el desarrollo de la Región Metropolitana. Resumen Ejecutivo Fondart Folio 215947

REYES-PAECKE, S. y FIGUEROA I. (2010). Distribución, superficie y accesibilidad de las áreas verdes urbanas en Santiago de Chile. EURE 36, 89-110.

TYRVÄINEN, L., PAULEIT, S., SEELAND, K. y DE VRIES, S. (2005). Benefits and uses of urban forests and trees. In: Konijnendijk, C.C., Nilsson, K., Randrup, T.B. y Schipperijn, J. (Eds.), Urban Forests and Trees. Springer, Berlin, pp. 81-114

WANG, XJ. (2009). Analysis of problems in urban green space system planning in China. Journal of Forestry Research 20: 79-82. 